\title{
The molecular electron density distribution. Meeting place of X-ray diffraction and quantum chemistry intermediate between theory and experiment
}

\section{Dirk Feil}

Chemical Physics Laboratory, University of Twente, PB 217, 7500 AE Enschede (Netherlands)

(Received 6 June 1991)

\begin{abstract}
Quantum chemistry and the concepts used daily in chemistry are increasingly growing apart. Among the concepts that are able to bridge the gap between theory and experimental practice, electron density distribution has an important place. The study of this distribution has led to new developments in theory, including Hellmann-Feynman theory and the density functional theory. The possibilities and limitations of these methods are discussed. Various ways of analysing the electron density distribution are presented and discussed. X-ray diffraction enables us to "observe" the electron density distribution and electrostatic properties. Experimental results are compared with the results of quantum chemical calculations. It is shown that even intermolecular interaction is observable with this method. Problems in determining ionic charges are seen to be inherent in the method.
\end{abstract}

\section{INTRODUCTION}

Chemistry, defined as the art of making compounds out of other compounds, has been a part of our culture from the early beginnings of mankind. Over the centuries much experience was accumulated and theories were developed to order this experience. Various cultures had different paradigms into which the theories had to fit, but in due course chemistry as we know it replaced all other theories.

It is generally believed that chemistry began to go in the right direction when Boyle revived the use of atomic theory in science and wrote his book The Skeptical Chymist in 1661. It was another century and a half before Dalton proposed his atomic theory in which compounds are considered to be formed by the union of the atoms of different elements. Although Newton had searched for the forces between atoms, by the time of Dalton no physical forces had been discovered that seemed to be capable of keeping atoms together. Consequently a new concept, valency, was introduced to account for bonding. With this new 
concept began the divorce of chemistry from physics. In the course of the nineteenth century chemistry developed rapidly and the covalent bond between atoms in molecules became a widely used, but little understood, concept. A wide variety of properties was assigned to atoms by chemists, quite contrary to the notion of simplicity that ought to characterize these fundamental building blocks of matter according to the physicists [1]. The gap between chemistry and physics was so wide that many scientists denied the existence of atoms and molecules. The discovery of X-ray diffraction by von Laue and the application of the method to the elucidation of the structure of compounds by Bragg and co-workers brought a definite end to the discussion. Nevertheless the gap remained and is illustrated by Lewis' electronic theory of valence (1916) which became widely adopted by chemists. Immediately after the publication of this theory in 1916, Debye tried to show the presence of two electrons in the bonding region of the covalent bonds in diamond, in which he used his recently developed powder method to obtain the diffraction intensities [2]. His failure to detect the electron pair is in agreement with present-day quantum chemistry and with accurate single-crystal measurements. Fortunately for the Lewis model, the discrepancy between theory and experiment did not become known among chemists and the model has remained in use up to the present day. Soon afterwards came the quantitative theory of quantum mechanics and covalent bonding was no longer a mystery to physicists. This led Dirac in 1929 to make his famous statement that "... the underlying physical laws necessary for the mathematical theory of a large part of physics and the whole of chemistry are thus completely known..." [3]. More recently, Ballhausen [4] remarked that "today we realize that the whole of chemistry is one huge manifestation of quantum phenomena". These quotations express the general feeling that chemistry and physics are united again.

Only a few people worry about the wide gap between the concepts used in the organic laboratory and the "beyond Hartree-Fock" activities of the quantum chemists [5]. Again the gap between theory used in computations and the qualitative concepts used in the laboratory seems to widen. Against this background one has to place the search for concepts that originate from (advanced) theory and are of practical value in the daily life of chemists.

The electron density distribution seems to be such a concept. It is rigorously defined as

$$
\begin{aligned}
\rho(\mathbf{r})=n \int & \Psi^{*}\left(\mathbf{r}_{1}=\mathbf{r}, \sigma_{1}, \mathbf{r}_{2}, \sigma_{2}, \ldots \mathbf{r}_{n}, \sigma_{n}\right) * \\
& \Psi\left(\mathbf{r}_{1}=\mathbf{r}, \sigma_{1}, \mathbf{r}_{2}, \sigma_{2}, \mathbf{r}_{n}, \sigma_{n}\right) \mathrm{d} \sigma_{1} \mathrm{~d} \mathbf{r}_{2} \mathrm{~d} \sigma_{2} \ldots \mathrm{d} \mathbf{r}_{n} \mathrm{~d} \sigma_{n}
\end{aligned}
$$

The wavefunction $\Psi$ describes the state of the $n$ electrons of the system. Nuclear positions appear as variables (in which case we have to integrate over them) or as parameters (implying the use of the Born-Oppenheimer approx- 
imation). As a function in three dimensions, the electron density distribution is not difficult to visualize.

Over the course of time, the electron density distribution acquired some standing due to a number of theorems. Hellmann [6] and Feynman [7] showed using quantum mechanics that the forces on the nuclei in a molecule can be calculated by classical electrostatic theory when $\rho(\mathbf{r})$ is interpreted as the density of a charge cloud. The corpuscular nature of the electron does not seem to play any role at all. In the following we briefly discuss the use and the limitations of the Hellmann-Feynman theorem. More recently, Hohenberg and Kohn [8] showed that for the ground state, energy is a unique functional of the electron density distribution. It seems remarkable that a property that requires the calculation of the two-particle density matrix is fully contained in the oneparticle density. On the basis of this theorem, Kohn and Sham [9] were able to derive a new method for calculating the electronic structure of atoms, molecules and crystals. Unfortunately the Hohenberg-Kohn theorem proves the existence of the functional, but not its form. Consequently, Kohn and Sham had to make assumptions in order to obtain concrete results. We show later in this paper that this method can yield valuable results.

Analysis of the electron density distribution in the hydrogen molecule shows that the formation of the covalent bond is accompanied by an increase in the electron density in the bonding region, a result that is consistent with the Hellmann-Feynman theorem. This increase can be observed in covalent carbon bond as well, but seems to be largely absent in many oxygen containing bonds. Bader showed that the type of bond can be better derived from an analysis of the Laplacian of the electron density distribution of the bonds.

Molecular biology shows that electrostatic interaction plays a major role in the world of living matter. The ubiquitous hydrogen bond has as a major component the electrostatic attraction between the positive hydrogen atom and the negative acceptor atom, but other examples abound. The other major type of molecular interaction, the London or dispersion interaction, is based on the correlation between electrons on the interacting monomers. To a large extent the London interaction can be calculated from transferable atom-atom interactions, restricting the necessary molecular information to geometrical parameters. The calculation of electrostatic interaction, however, requires knowledge of the electron density distribution in the monomers, a property that is difficult to obtain, both experimentally and theoretically, for molecules that consist of more than a few atoms. Within certain groups of compounds it is possible to partition the electron density distribution into atomic fragments in such a way that these fragments can be used to construct other molecules. Various ways of partitioning exist and these are discussed later.

We also report in this paper on the possibility of obtaining the necessary information from X-ray diffraction. The results can be compared with the results of quantum chemical calculations. As these calculations suffer from a 
number of approximations, discrepancies can originate from either the experimental or the theoretical values of the quantities being compared. Some of the main problems associated with this procedure are discussed.

\section{HELLMANN-FEYNMAN THEOREM}

Most textbooks on quantum chemistry show with a simple derivation that exact wavefunctions satisfy the Hellmann-Feynman theorem

$\frac{\mathrm{d} E}{\mathrm{~d} Q}=\left\langle\Psi\left(\frac{\mathrm{d} H}{\mathrm{~d} Q}\right) \Psi\right\rangle$

in which $Q$ is any parameter of the system concerned.

At first sight it seems a powerful theorem which allows the calculation of the derivative of the energy with respect to any parameter in one calculation. The most important example is the derivative of the energy with respect to one of the nuclear position parameters $\mathbf{R}, \partial E / \partial \mathbf{R}$, yielding the force on the particular nucleus. By assuming the electron density distribution to represent an inhomogeneous charge cloud, the force on a particular nucleus can be calculated in a completely classical way. This is known as the electrostatic theorem.

Originally the theorem was derived for exact wavefunctions. Hurley [10] was able to derive conditions under which the theorem holds also for an approximate wavefunction. The Hartree-Fock (HF) method satisfies these conditions, but application of the linear combination of atomic orbitals (LCAO) expansion requires the use of basis functions that do not automatically move with the nuclear positions.

\section{TABLE 1}

Forces (in a.u.) on the atoms in the LiH molecule based on HF calculations using a large basis set $^{a}$

\begin{tabular}{|c|c|c|c|c|c|}
\hline \multirow{2}{*}{$\begin{array}{l}\mathrm{Li}-\mathrm{H} \\
(\AA)\end{array}$} & \multirow{2}{*}{$\begin{array}{l}\text { Energy } \\
\text { (a.u.) }\end{array}$} & \multicolumn{2}{|l|}{ Lithium } & \multicolumn{2}{|l|}{ Hydrogen } \\
\hline & & $F_{\text {tot }}$ & $F_{\mathrm{H}-\mathrm{F}}$ & $F_{\text {tot }}$ & $F_{\mathrm{H}-\mathrm{F}}$ \\
\hline 1.58 & -7.9803910 & -0.0056 & 0.1689 & 0.0056 & 0.0066 \\
\hline 1.60 & -7.9805509 & -0.0029 & 0.1670 & 0.0029 & 0.0038 \\
\hline 1.61 & -7.9805922 & -0.0015 & 0.1704 & 0.0015 & 0.0024 \\
\hline 1.62 & -7.9806089 & -0.0002 & 0.1708 & 0.0002 & 0.0011 \\
\hline 1.63 & -7.9806019 & 0.0010 & 0.1711 & -0.0010 & -0.0002 \\
\hline 1.64 & -7.9805719 & 0.0022 & 0.1714 & -0.0022 & -0.0014 \\
\hline 1.66 & -7.9804461 & 0.0044 & 0.1718 & -0.0044 & -0.0037 \\
\hline 1.70 & -7.9799516 & 0.0085 & 0.1721 & -0.0085 & -0.0079 \\
\hline
\end{tabular}

${ }^{a} \mathrm{Li},(11 \mathrm{~s} 4 \mathrm{p}) /[5 \mathrm{~s} 3 \mathrm{p}] ; \mathrm{H},(8 \mathrm{~s} 3 \mathrm{p} 1 \mathrm{~d}) /[4 \mathrm{~s} 3 \mathrm{p} 1 \mathrm{~d}][37] . F_{\text {tot }}$ is calculated from the energy, while $F_{\mathrm{H}-\mathrm{F}}$ is based on the electrostatic theorem. 
Even the use of very accurate wavefunctions does not guarantee that the calculation of the force on a particular nucleus from the electron density distribution will yield the same result as the derivative of the energy with respect to nuclear displacement as can be shown by the example of the $\mathrm{LiH}$ molecule (G. Velders, personal communication, 1991). The forces on each of the two atoms, calculated from the electron density cloud, for different interatomic distances are given in Table 1 . The equilibrium distance can be derived from the energy of the system. The calculations are carried out for a very extended basis set. The Hellmann-Feynman or electrostatic forces on the two atoms are not the same in magnitude as they should be. Enlargement of the basis set improves the values for the hydrogen atom, but the results for the lithium atom remain bad. It is clear that the theorem cannot be used to obtain forces in a routine way. Nevertheless, much qualitative reasoning has been based on the theorem [11].

\section{DENSITY FUNCTIONAL THEORY}

Based on Hohenberg and Kohn's theorem, Kohn and Sham derived an equation for orbitals to be occupied by non-interacting electrons. They showed that the electron density distribution obtained by summing the square of the orbitals is the same as the electron distribution of the pertaining atom or molecule in the ground state

$\rho(\mathbf{r})=\sum_{i} \phi_{i}^{2}$

These orbitals satisfy the Kohn-Sham equation

$\hat{F} \phi_{i}=\epsilon \phi_{i}$

The operator $\hat{F}$ closely resembles the Fock operator and can be obtained from it by replacing the exchange operator $\hat{K}$ by the exchange-correlation operator $\hat{V}_{x c}$ of the Kohn-Sham theory. Kohn and Sham showed this operator to be a potential whose value at a particular position depends on the total electron density distribution in the system. They could only give an approximate form for it. A widely employed approximation is the local density approximation in which $\hat{V}_{\mathrm{xc}}$ is given by

$\hat{V}_{\mathrm{xc}}(\mathbf{r})=-3 \alpha\left(\frac{3}{8 \pi} \rho(\mathbf{r})\right)^{1 / 3}$

The factor $\alpha$ was originally assumed to be 1 , but $2 / 3$ seems to be better. Some people fix its value by empirical methods. The final equation closely resembles a Fock equation in which the exchange potential is replaced by an expression given by Slater. Consequently, the method is often called the Hartree-FockSlater (HFS) method.

Baerends et al. [12] developed a LCAO version of this method, which has the advantage that the computing effort increases with the number $(N)$ of 
basis functions as $N^{3}$ instead of $N^{4}$ as is the case with HF calculations. As the method allows the use of the efficient Slater-type orbitals (STOs) rather large basis sets can be used. Krijn et al. [13] extensively studied the quality of the electron density distributions calculated with this method. A comparison with the HF method for water is shown in the next section.

\section{QUANTUM CHEMICAL CALCULATIONS OF THE ELECTRON DENSITY DISTRIBUTION}

The electron density distribution is a one-particle property and thus the HF method should yield good values of this quantity. There are several ways to judge the quality of the electron density distribution. The most widely used method is to compare the various moments of the distribution with reference values obtained from measurements or from other calculations. Another way is to look at plots of the distribution in various cross-sections of the system. We shall give examples of both for the water molecule.

Figure 1(C) shows the deformation density distribution,i.e. the difference between the electron density distribution in the molecule and that in the promolecule. The promolecule is defined as the linear combination of undisturbed, spherical atoms, positioned at the nuclear centres of the molecule.

The calculations were carried out using the HF method, employing various basis sets. Using the $6-31 G^{* *}$ basis set as reference (Fig. $1(\mathrm{C})$ ), the influence of omission of polarization functions can be shown (Fig. 1(B)). The effect of a further reduction of the basis set is seen to be relatively small (Fig. 1(A)). Extension of the basis set beyond 6-31G** shows minor changes in the contours (Fig. 1(D)). The extensive studies of Rosenberg and Shavitt [14] and of Bawagan et al. [15], however, show that inclusion of diffuse functions have considerable influence on the value of the dipole moments of the molecule. The same result is obtained by extending the basis set in a HFS calculation. (L.G. Poorthuis and D. Feil, personal communication, 1991) (Table 2). The importance of using a large basis set in calculating electrostatic properties cannot be overstated.

Correlation has only a minor influence on the electron density distribution. Rigorous quantum chemical calculations invoking full configuration interaction (CI) are not feasible for anything other than the smallest molecules. Restriction to singly and doubly excited states extends the range of systems that

Fig. 1 (opposite). The effect of basis set and correlation on the deformation density of water. The deformation density obtained with the $6-31 G^{* * *}$ basis set is used as reference. (A) 4-31G, (B) 631G, (D) triple zeta; complemented with a double set of polarization functions. The deformation density has been subtracted from the deformation density obtained with the $6-31 \mathrm{G}^{*+}$ basis set. Contour interval $0.05 e \AA^{-3}$. (C) Deformation density with the $6-31 \mathrm{G}^{* *}$ basis set. Contour interval $0.1 e \AA^{-3}$. (E) Correction of the $6-31 G^{* *}$ results for correlation, calculated with the MP2 method. Contour interval $0.01 e \AA^{-3}$. 

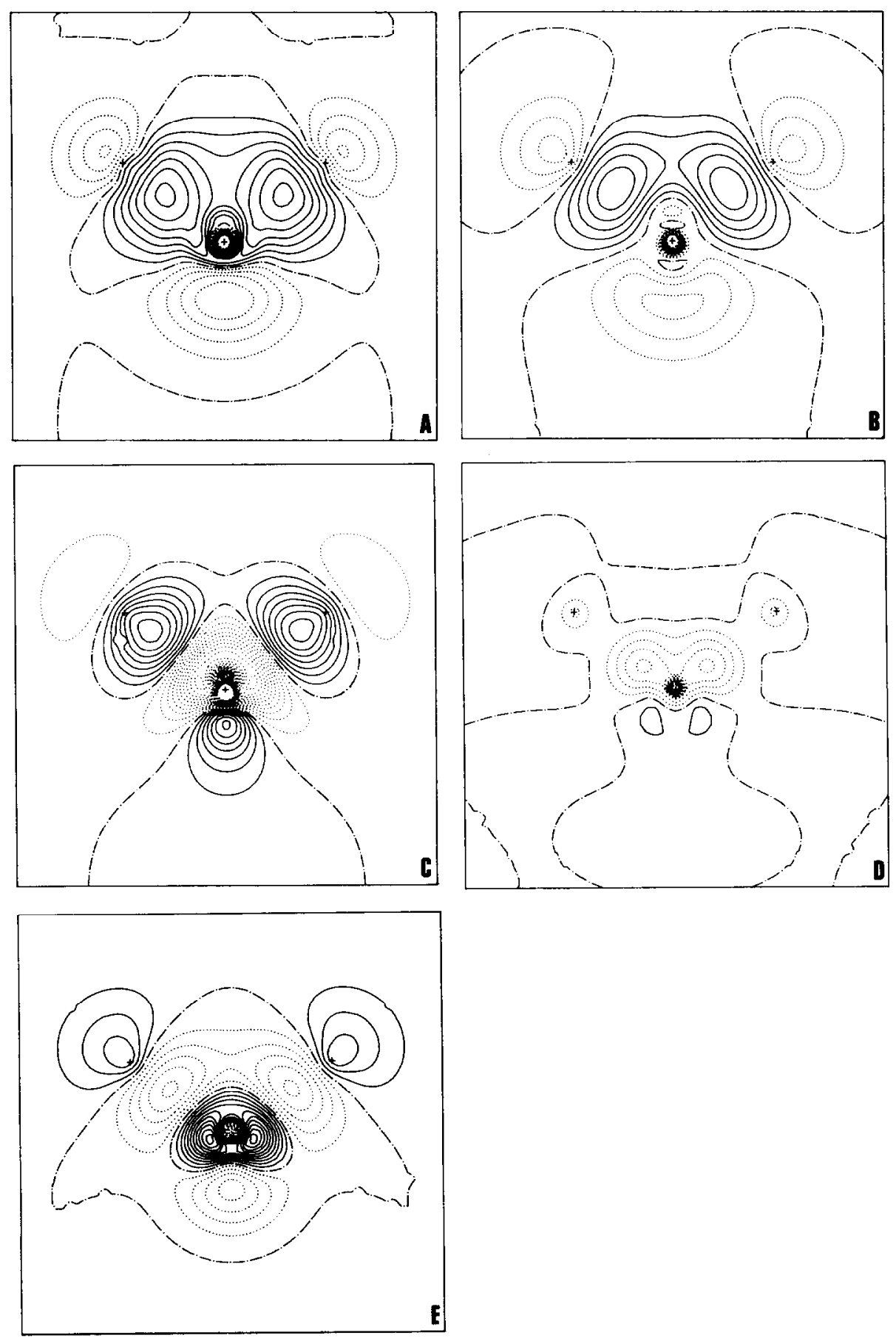

Fig. 1. 


\section{TABLE 2}

The static dipole moment of the water molecule, calculated using the HFS method, employing different basis sets

\begin{tabular}{lll}
\hline Basis set & STOs & $\begin{array}{l}\text { Dipole moment } \\
\text { (a.u.) }\end{array}$ \\
\hline $\mathrm{DZ}$ & $5 \mathrm{~s}, 2 \mathrm{p} / 2 \mathrm{~s}$ & 1.033 \\
$\mathrm{DZD}^{\mathrm{a}}$ & $5 \mathrm{~s}, 2 \mathrm{p}, \mathrm{d} / 3 \mathrm{~s}, \mathrm{p}$ & 0.765 \\
$39-\mathrm{STO}^{\mathrm{b}}$ & $5 \mathrm{~s}, 4 \mathrm{p}, 2 \mathrm{~d} / 3 \mathrm{~s}, \mathrm{p}$ & 0.752 \\
Extended $^{\mathrm{a}}$ & $8 \mathrm{~s}, 4 \mathrm{p}, \mathrm{d}, \mathrm{f} / 3 \mathrm{~s}, 2 \mathrm{p}, \mathrm{d}$ & 0.728 \\
& & 0.730 (exp.)
\end{tabular}

Krijn and Feil [19].

bosenberg and Shavitt [14].

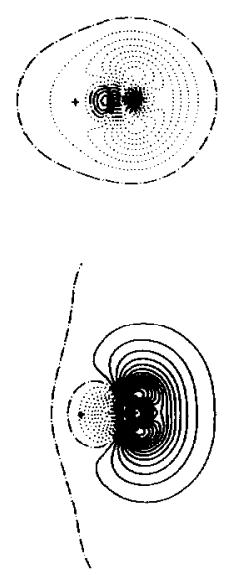

$\mathrm{H}_{2} \mathrm{O}$
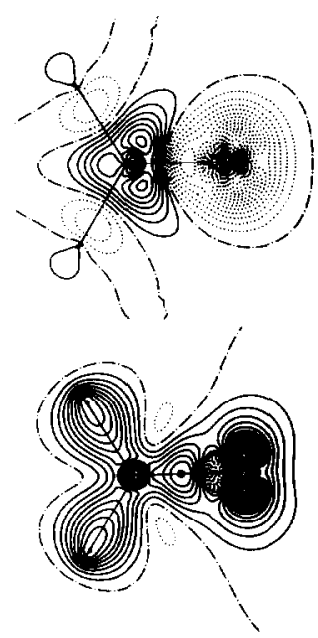

$\mathrm{H}_{2} \mathrm{CO}$
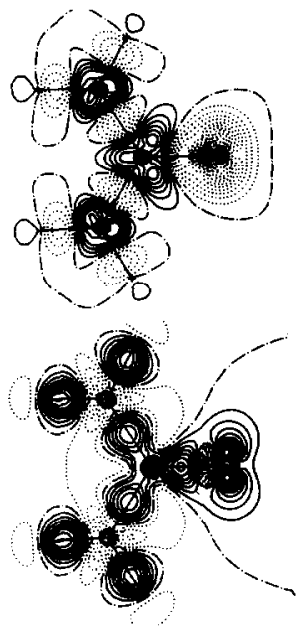

urea

Fig. 2. The deformation density and the correlation correction for a number of oxygen-containing molecules. The results for water (in the plane this bisects the molecule), formaldehyde and urea are shown. The contour interval in the deformation densities (lower row) is $0.1 e \AA^{-3}$ and in the correlation density (upper row) $0.01 e \AA^{-3}$.

can be handled, but it introduces the problem of size-consistency, a phenomenon in which the introduction of a non-interacting replica of the molecule under consideration into the system does not always double the energy as it should. The Møller-Plesset (MP) perturbation method satisfies the size-consistency requirement. The work of Wang and Boyd [16] shows that the corrections to the electron density distribution calculated with the second-order 


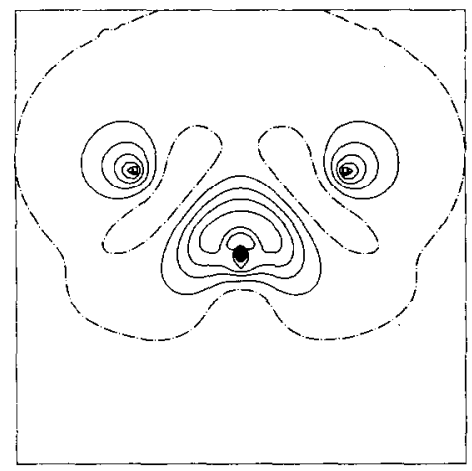

Fig. 3. The difference between the deformation density distributions calculated with the HFS and the HF methods. The bases used were TZD and 6-31G**, respectively. The contours are drawn at $0,+0.0005,+0.0015,+0.0035,+0.0075$ a.u., etc. Positive contours are indicated by solid lines.

MP method (MP2) are very similar to those obtained by means of a CI calculation. Figure 1(E) shows the results of an MP2 calculation on the water molecule.

Assuming that the MP2 correction accounts for most of the correlation, we calculated the correlation density, defined as the correction to the electron density distribution by including correlation in the calculations, for a number of oxygen containing molecules [17]. It can be seen from Fig. 2 that the correlation density in the region of the oxygen atom is very similar, suggesting transferability.

We now turn to the HFS method. The method has been shown to yield very good values for the properties of a number of molecules $[18,19]$. Nevertheless, the electron density distribution obtained with this method for the water molecule is somewhat different from the one calculated using the HF value, even when correlation is taken into account. It is intriguing that the HFS method gives better values for the permanent dipole moment and the linear polarizabilities. The major part of the difference is due to the difference in the density in the promolecule. For many purposes this difference is of no interest and we show the difference in the deformation density calculated with the two methods (Fig. 3). Comparison of Fig. 3 with Fig. 1 shows that the difference exceeds the correlation density.

A final note of warning is necessary. The HF method with two electrons per orbital, the so-called restricted Hartree-Fock (RHF) method, does not give a proper description of the molecule in the process of dissociation. Consequently, RHF calculations are not suitable for calculating transition states.

ANALYSIS OF THE ELECTRON DENSITY DISTRIBUTION

The Laplacian

Neither the electron density distribution nor the deformation density dis- 
tribution yield much information that can be related to the classical chemical concepts. The deformation density distribution indicates where the electrons go and where they come from when bonds are formed, but covalent bonds in one and the same molecule may have a widely different appearance. This is well illustrated by the furan molecule where the bonds involving oxygen atoms show less piling up of charge than that which is seen in the other bonds. This effect has been observed before and has been discussed by Dunitz and Seiler [20]. More information can be obtained after some simple mathematical treatments. The subject has been dealt with fully by Bader [21] and only some major points are discussed here. The electron density distribution in a molecule varies considerably in the core regions of the atoms to become a relatively smooth function in the interatomic regions. The gradient $\nabla \rho(\mathbf{r})$ of the electron density distribution turns out to play an important role in a number of theories. Allen et al. [22] have shown that the dissociation energy of a large number of diatomic molecules is closely related to the inhomogeneity kinetic energy $T_{2}$ defined as

$T_{2}=\frac{h}{72 m} \int \frac{(\nabla \rho)}{\rho} \mathrm{d}^{3} \mathbf{r}$

The dissociation energy, divided by the square of the number of electrons in the molecule, is seen to decrease linearly with increasing $T_{2}$.

Bader investigated trajectories traced out by the vector $\nabla \rho(\mathbf{r})$, the gradient paths. Points in space where the gradient is zero, the so-called critical points, are important in defining atoms in molecules. The next derivative $\nabla^{2} \rho(\mathbf{r})$, the Laplacian, shows highly interesting features. Barely observable shoulders in the $\rho(\mathbf{r})$ curve turn into sharp peaks in the representation of $-\nabla^{2} \rho(\mathbf{r})$. This function has values in the bonding region that give a good indication of the type of bond between the atoms involved. This is illustrated in Table 3 .

We now return to the furan molecule, where it can be seen that the $-\nabla^{2} \rho(\mathbf{r})$

\section{TABLE 3}

Characterization of interatomic interaction by the electron density and its Laplacian at the critical point in the interatomic region (all values in a.u.)

\begin{tabular}{llr}
\hline Molecule & $\rho(\mathbf{r})$ & $\nabla^{2} \rho(\mathbf{r})$ \\
\hline $\mathrm{H}_{2}$ & 0.27 & -1.38 \\
$\mathrm{~N}_{2}$ & 0.72 & -3.05 \\
$\mathrm{O}_{2}$ & 0.55 & -1.01 \\
$\mathrm{NaCl}$ & 0.03 & 0.20 \\
$\mathrm{NaF}$ & 0.05 & 0.47 \\
$\mathrm{MgO}$ & 0.09 & 0.65 \\
\hline
\end{tabular}



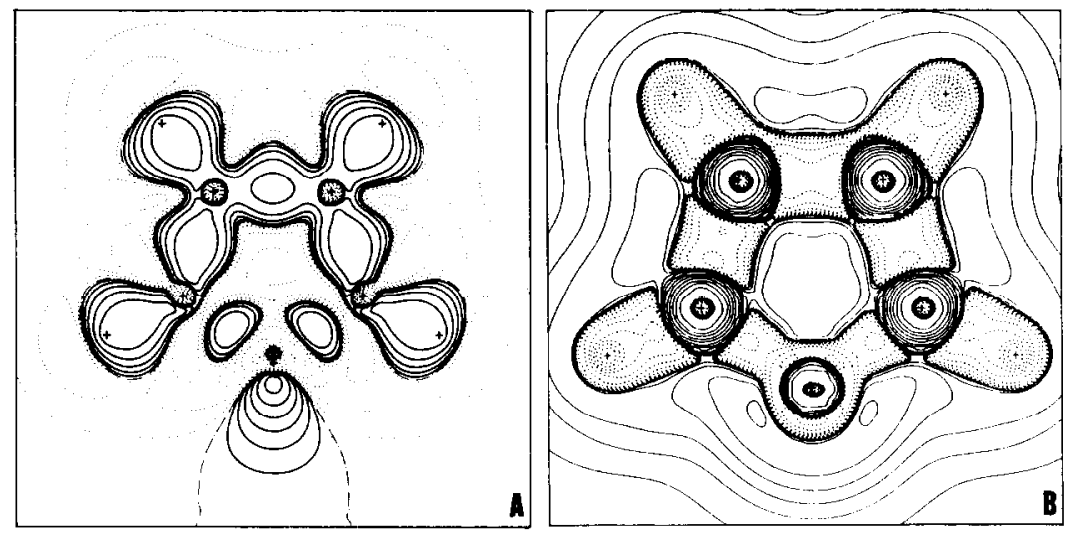

Fig. 4. The electron density distribution and its Laplacian for furan, based on a Hartree-Fock calculation employing a $6-31 \mathrm{G}^{* *}$ basis set. (A) Deformation density distribution; contours at 0 , $+0.002,+0.004,+0.008$ a.u., etc. (B) Laplacian of the deformation density; contours at $0,+0.01$, $+0.02,+0.04$ a.u., etc. Dashed lines are negative contours.

distribution in the bonding regions shows values that are expected of covalent bonds (Fig. 4).

In regions of space where the Laplacian is negative, the potential energy dominates the local total energy. Where the Laplacian is positive, the kinetic energy is in local excess [23].

\section{Potential}

In the theory of chemical reactivity the potential distribution beyond the van der Waals surface of the molecule plays an important role. This distribution can easily be calculated from the electron density distribution with the help of the integrated Poisson equation

$V(\mathbf{r})=\left(4 \pi \epsilon_{0}\right)^{-1} \int \frac{\rho(\mathbf{r})^{\prime}}{\left|\mathbf{r}-\mathbf{r}^{\prime}\right|} \mathrm{d}^{3} \mathbf{r}^{\prime},+V_{\mathrm{N}}(\mathbf{r})$

in which $V_{\mathrm{N}}(\mathbf{r})$ is the potential due to the nuclei.

Since the potential is obtained by integration over the molecular volume, the result seems to be less vulnerable to errors due to inadequate basis sets and the neglect of correlation. In hydrogen bonding we find a geometry that allows regions of positive $V(\mathbf{r})$ belonging to one molecule to be superimposed on regions of negative potential due to the other partner in the bond.

\section{Partitioning}

Whereas subtle features in the electron density distribution of the intera- 
tomic regions inform us about the type of bonding between atoms, the distribution of charge over the atoms of a molecule plays an important role in the electrostatic interaction between molecules. The usually uneven distribution leads to an inhomogeneous potential distribution on the molecular surface. This potential distribution can be calculated directly from the molecular wavefunction. Another approach is to divide the molecular charge distribution into atomic charge distributions, calculate the various moments of the local distributions and derive the potential from these moments [24]. In general, moments up to second order (the quadrupole moments) are needed to obtain more or less satisfactory values at the molecular surface. The method implies that the molecular charge distribution can be partitioned into atomic fragments. Several methods are available for doing this and the most important ones are discussed here briefly. Bader devised a method which yields atoms that show highly interesting characteristics. He chose as boundaries the planes where the flux of the electron density distribution vanishes

$\nabla \rho(\mathbf{r}) \cdot n(\mathbf{r})=0$

in which $n(\mathbf{r})$ is the normal to the boundary at $\mathbf{r}$. With these boundaries he was able to show that the viral theorem applies to the individual atoms [25]. Unfortunately, the calculation of the various moments of these atoms requires considerable computational effort. Another disadvantage is the irregular behaviour of the atomic scattering factor, owing to the sharp boundary of the atomic distribution [26]. Hirshfeld [27] has suggested the stockholder method which yields atoms with fuzzy boundaries. One first calculates the electron density distribution of the promolecule $\rho_{\mathrm{pm}}(\mathbf{r})$ and the electron density distribution of atom $\mathrm{A}$ in the molecule is then derived by applying the following operation to the molecular electron density distribution

$\rho_{\mathrm{A}}(\mathbf{r})=\frac{\rho_{\mathrm{A}}^{0}(\mathbf{r})}{\sum_{\mathrm{A}} \rho_{\mathrm{A}}^{0}(\mathbf{r})} \rho(\mathbf{r})$

where $\rho_{\mathrm{A}}^{0}$ is the density of the free atom A. The resulting atomic electron density distributions are transferable within groups of related molecules [27]. In the following section we show that the method is also applicable to experimental electron density distributions.

\section{X-RAY DIFFRACTION AND THE ELECTRON DENSITY DISTRIBUTION}

In his pioneering work on X-ray diffraction Debye showed that the technique can be used to obtain information on the electron structure of atoms and molecules in crystals [2]. Nowadays, structural factors can be obtained from diffraction intensities to an accuracy of about $2 \%$ [28]. Experience shows that the information on the electron density distribution which is contained in the diffraction intensities can be reproduced with a model that contains about 45 
parameters per atom [29]. Note that this refinement model itself does not contain any chemical or physical information that is specific to the crystal under study; the only requirement is sufficient flexibility to represent the electron density distribution with an accuracy that surpasses the experimental accuracy when the parameters are optimized. For the type of structures considered here we find that the number of data greatly exceeds the number of parameters to be determined. This allows a reduction in the amount of noise in the data at the expense of the introduction of only small systematic errors. Consequently, we assume that the structural factors based on the refined model are more accurate than the measured ones, i.e. we assume an accuracy of about $1-1.5 \%$. In the following the measured electron density distribution is the one that is calculated with the structural factors based on the refined model.

Because the electron density distribution of the promolecule changes only slightly when the atoms form bonds, the corresponding changes in the structure factors amount to a few percent of the structural factors themselves. This is demonstrated in Fig. 5, where a cross-section of the Fourier transform of the deformation density of $\mathrm{N}_{2}$ is shown. As the molecules in a crystal execute thermal motion, the electron density distribution is smeared and the values of the transform must be multiplied by a temperature factor.

At low scattering angles $(\theta)$ the $\mathrm{X}$-rays sample the outer regions of the atoms, where the diffuse density barely contributes to the scattering. At high scattering angles the inner atomic regions, where positive and negative deformation density alternate, are probed. Thermal motion causes the superposition of the positive and the negative regions, resulting in an average structure of the inner region with vanishing deformation density. Since elastic scattering is determined by the thermally averaged electron density distribution, the

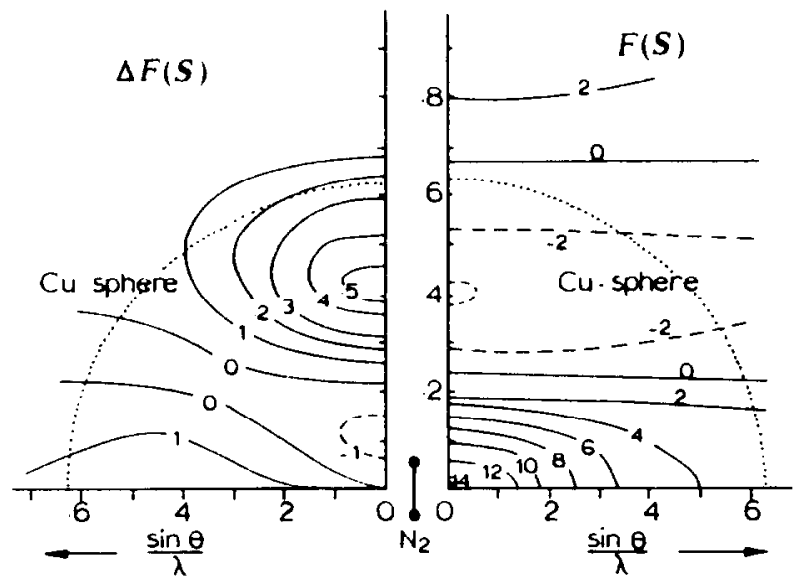

Fig. 5. The Fourier transform, $F(S)$, of the electron density distribution and the transform of the deformation density, $\Delta F(S)$, of the $\mathrm{N}_{2}$ molecule. 
structural factors are sensitive to bonding features in the interatomic regions of the molecule, but do not contain information on either the inner atomic or the outer molecular regions.

Coppens [30] used this sensitivity for the determination of the occupancy of the d orbitals in the transition-metal complexes such as iron phthalocyanine where various theoretical approaches give different answers. More often the method is used to verify quantum chemical calculations, in particular to test new and existing approximations. As an example we compare the results of a quantum chemical calculation of the thermally averaged deformation density with the results obtained from the X-ray diffraction study of imidazol (Fig. 6). The molecule is shown in Fig. 7 and details of the measurements are given in Table 4.

Note the close resemblance between the two sets of results in Fig. 6. Some of the differences between the two are due to errors in the measurements or to the approximations in the quantum chemical model. Other differences are the result of comparing a molecule in a crystal with a free molecule.

We now compare the potential due to the molecule in the crystal, based on diffraction data, with the one obtained from an HF calculation (Fig. 7). The pronounced negative region next to the nitrogen atom is the obvious choice for a hydrogen bond, an expectation that is confirmed by the crystal structure.

The work by Krijn et al. [31] on oxalic acid dihydrate shows that it is possible to push the usefulness of diffraction for the study of the electron density distribution further. Mutual polarization of the molecular charge distributions in oxalic acid dihydrate occurs in particular in the region of the hydrogen bond. This interaction density, defined as the difference between the density in the
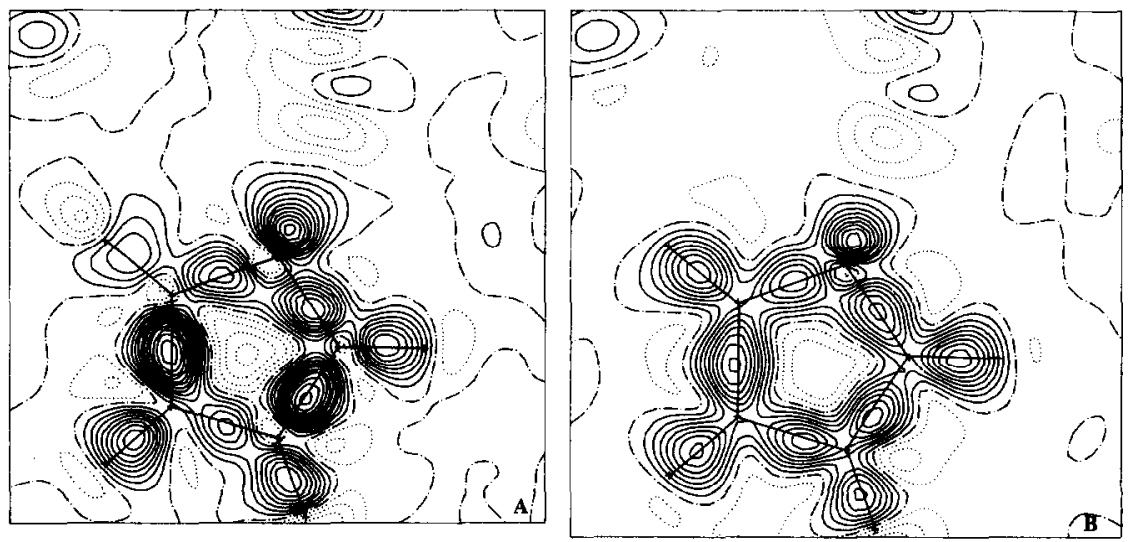

Fig. 6. The thermally averaged deformation density in imidazol. (A) Experimental. (B) Theoretical, based on the molecular deformation density, calculated with the HFS method, employing a double zeta basis, supplemented with polarization functions on all atoms. Contour interval $0.05 e$ $\AA^{-3}$. Dashed lines are negative contours. 

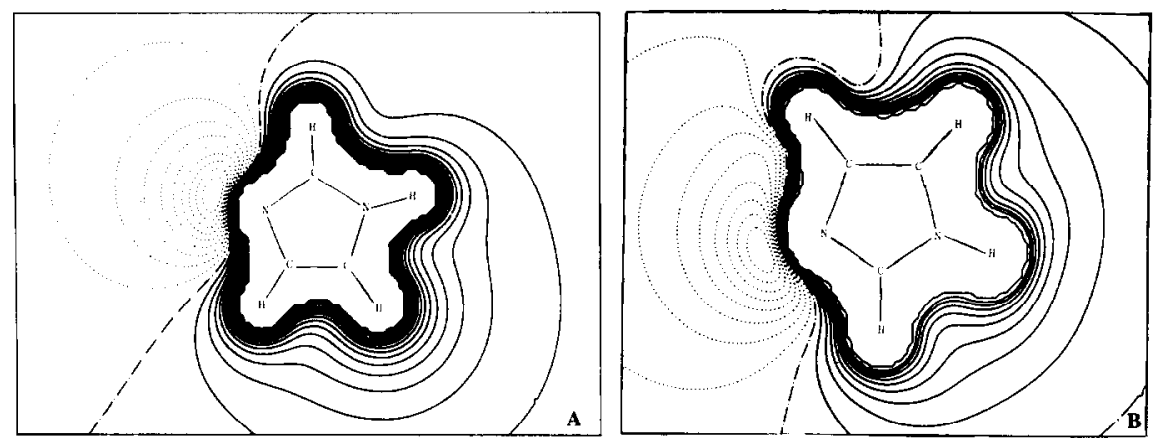

Fig. 7. The potential distribution around imidazol according to theory and experiment. (A) Based on the electron density distribution calculated with the HF method, employing a 6-31G $\mathrm{G}^{* *}$ basis set. (B) Based on the molecular electron density distribution as observed with $\mathrm{X}$-ray diffraction. Contour interval $0.025 e \AA^{-3}$. Dashed lines are negative contours.

\section{TABLE 4}

Details of the measurements on imidazol

Space group $P Q_{1} / \mathrm{c}$

$a=7.5750(2)$

$b=5.3730(2)$

$c=9.8140(22)$

$\cos \beta=-0.48900$

4 molecules per unit cell

Diffractometer PW1100

$100 \mathrm{~K}$

$(\sin \theta / \lambda)_{\max }=1.2$

Measured reflections

15921

Unique reflections

3663

$I>3 \sigma(I)$

2723 , internal R $3 \%$

Multipole refinement

$R=1.3 \%, R_{\mathrm{w}}=1.8 \%$

crystal and that in the procrystal, can be "observed" by subtracting the superposition of the (calculated) molecular density distributions from the experimental one [31] (Fig. 8(A)). When the interaction is included by performing a supermolecule calculation, the difference between experiment and theory in the interaction region disappears, leaving mainly experimental noise (Fig. 8(B)).

Recently, Bruning [32] studied the observability of a number of quantities related to the electron density distribution. He first calculated the electron density distribution in the crystal of magnesium formate dihydrate with the HFS-method employing an extensive basis set. Subsequently, the structural factors were calculated, assuming the same thermal motion as was observed in 


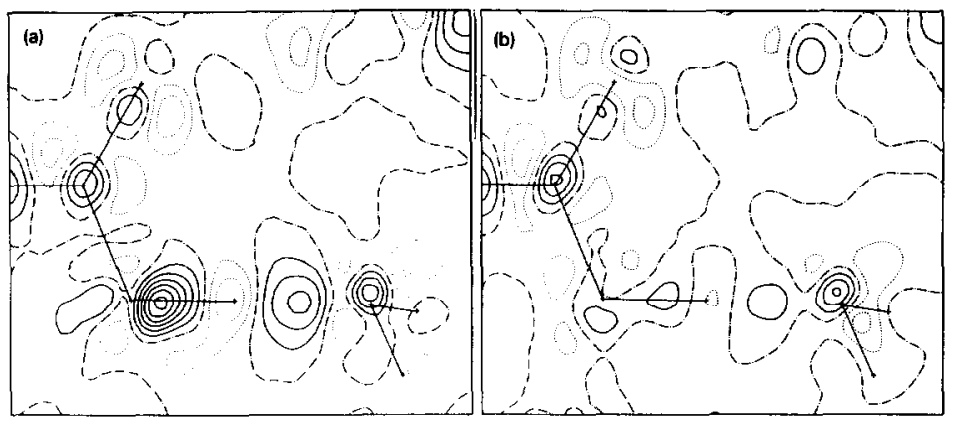

Fig. 8. The interaction density distribution in oxalic acid dihydrate. The difference between the experimental density distribution and the one based on HFS calculations of $(A)$ the separate molecules involved, without interaction, and (B) the supermolecule, consisting of interacting molecules. The thermally averaged system is shown; contour interval $0.05 e \AA^{-3}$.
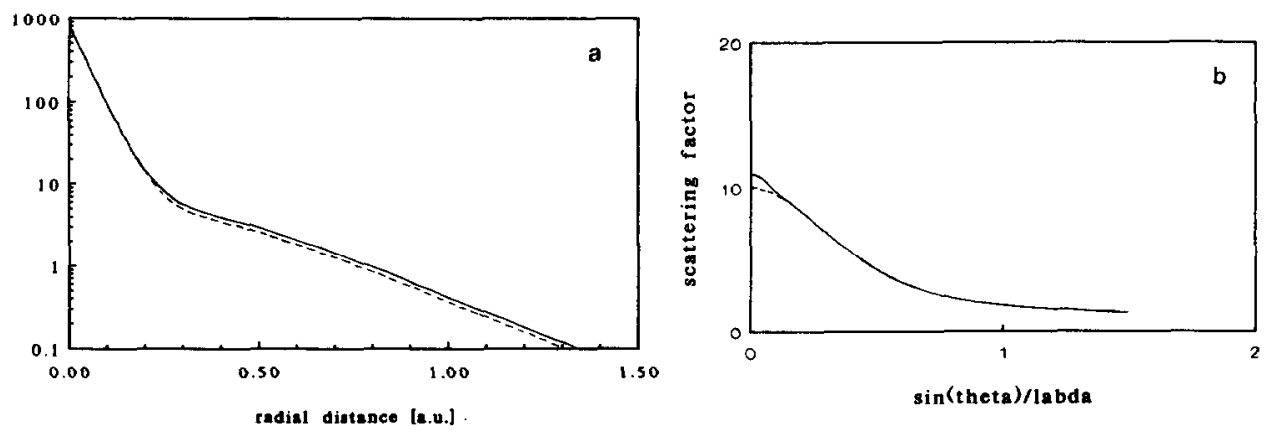

Fig. 9. The sodium atom. (A) The electron density as a function of distance to the radius. (B) the scattering factor as a function of the scattering angle.

an X-ray diffraction study of the compound. Employing the widely used refinement model of Hirshfeld [29] an analytical description of the electron distribution was found. The structural factors of the refined model deviate only a few tenths of a percent from the ones calculated directly from the quantum chemical model. Since there are, by definition, no random errors in the original electron density distribution, this slight discrepancy is due to the inflexibility of the model. The discrepancy is only a small fraction of the noise in experimental data. On partitioning the resulting density distribution, the atomic fragments carry charges that differ considerably from those derived from the original density distribution by the same partitioning method. In particular the charge on the magnesium atom is difficult to reproduce, confirming the objections raised by Bragg et al. [33] in connection with Debye's work on the ionic charges in KF [2]. The physical basis of the difficulties is easy to understand if the electron density distribution and the scattering power of an ion are compared to those of the corresponding neutral atom (Fig. 9). 
TABLE 5

The electrostatic interaction (in $\mathrm{kJ} \mathrm{mol}^{-1}$ ) between urea molecules in the urea crystal ${ }^{\mathrm{a}}$

\begin{tabular}{lll}
\hline $\begin{array}{l}\text { Interacting } \\
\text { molecules }\end{array}$ & X-ray diffraction & Quantum chemistry \\
\hline $1-2$ & $-50(14)$ & -49.5 \\
$1-3$ & $-32(10)$ & -30.2 \\
$1-4$ & $-5(3)$ & -4.1 \\
$1-5$ & $+4(2)$ & +4.4 \\
$1-6$ & $+7(2)$ & +4.0 \\
$1-7$ & $+4(1)$ & +2.6 \\
\hline
\end{tabular}

${ }^{a}$ Molecule 1 is surrounded by molecules 2 and 3 in the first shell and by the others in the second shell. The values are calculated on the basis of the interaction between the atoms derived from Xray data and from $\mathrm{HF}$ calculations followed by partitioning [38].

Note that the differences only occur in the distant diffuse regions and, consequently, at low scattering angles. Unfortunately, low-angle-diffraction data are often affected by extinction, whereby X-rays are diffracted more than once in the crystal, violating the Born approximation on which the current kinematic theory is based. This is particularly the case in ionic crystals, thus preventing us from determining the charges on the atoms. The diffraction method cannot observe features much smaller than the wavelength used, so the inner electronic structure of the atom remains unknown. This problem is aggravated by the fact that elastic $\mathrm{X}$-ray diffraction observes the thermally averaged electron density distribution in which details are averaged out. This makes it highly unlikely that X-ray diffraction results can be used for the application of the electrostatic theorem. It is doubtful whether detailed analysis of the Laplacian of the experimental electron density analysis will yield relevant results in view of the effects of noise and thermal smearing. The same applies to the Bader charge analysis, where noise affects not only the value of the electron density, but also the boundaries of the atoms: both integrand and the limits of the integral are altered.

Excellent results are obtained with the application of the stockholder method on neutral molecules such as urea. Close agreement is found between the electrostatic interaction energies based on diffraction data and on $\mathrm{HF}$ calculations (Table 5).

Experimental stockholder charges were put to good use by Berkovitch-Yellin and Leiserowitz [34] in their analysis of the packing motifs of amides and carboxylic acids [35] and in the application of the Hartman theory of crystal growth [36].

\section{CONCLUSIONS}

Powerful computer programs exist to calculate accurate wavefunctions for 
small molecules. Comparison of the results obtained in this way with those obtained using approximate methods such as the HF method with restricted basis sets indicates the limited value of the latter. In particular, the calculation of the electron density distribution and closely related electrostatic properties such as the dipole moment and (hyper)polarizabilities requires the use of large basis sets and correction for correlation.

The study of the electron density distribution yields additional insight into the interaction between atoms and molecules. Relevant ways of partitioning the molecular electron density distribution into transferable atomic fragments exist.

$\mathrm{X}$-ray diffraction, supported by neutron diffraction, can be used to obtain electron density distributions of reasonable accuracy and sensitivity. The fact that the outer regions of atoms and molecules are not properly probed in this technique means that ionic charges cannot be determined. Partitioning of the charge of neutral molecules over the atomic fragments seems to be successful and has yielded useful results. Because elastic $\mathrm{X}$-ray diffraction is determined by means of the thermally averaged crystal structure, the detailed features of the core regions of atoms are washed away and the Hellmann-Feynman theorem cannot be applied.

\section{ACKNOWLEDGEMENT}

The help of Guus Velders and Gerrit Poorthuis in carrying out various calculations and in preparing several figures is gratefully acknowledged.

\section{REFERENCES}

1 W. Heisenberg, Das Naturbild der Heutigen Physik, Rowolt, Hamburg, 1955.

2 P. Debye and P. Scherrer, Phys. Zeitschr., 19 (1918) 474.

3 P.A.M. Dirac, Proc. R. Soc. London, Ser. A, 123 (1929) 714.

4 C.J. Ballhausen, J. Chem. Educ., 56 (1979) 357.

5 Z.B. Maksic (Ed.), Theoretical Models of Chemical Bonding, Part 1, Springer-Verlag, Berlin, 1990.

6 H. Hellmann, Einführung in die Quantenchemie, Deuticke, Leipzig, 1937, pp. 285-286.

7 R.P. Feynman, Phys. Rev., 56 (1939) 340.

8 P. Hohenberg and W. Kohn, Phys. Rev. B, 136 (1964) 864.

9 W. Kohn and L.J. Sham, Phys. Rev. A, 140 (1965) 1133.

10 A.C. Hurley, Proc. R. Soc. London, Ser. A, 226 (1954) 179, 193.

11 B.M. Deb, Rev. Mod. Phys., 45 (1973) 22.

12 E.J. Baerends, D.E. Ellis and P. Ros, Chem. Phys., 2 (1973) 41.

13 M.P.C.M. Krijn and D. Feil, Chem. Phys. Lett., 150 (1988) 45.

14 B.J. Rosenberg and I. Shavitt, J. Chem. Phys., 63 (1975) 2162.

15 A.O.Bawagan, C.E. Brion, E.R. Davidson and D. Feller, Chem. Phys., 113 (1987) 19.

16 L.-C. Wang and R.J. Boyd, J. Chem. Phys., 90 (1989) 1083.

17 G. Velders and D. Feil, in preparation. 
18 E.J. Baerends, P. Vernooys, A. Rozendaal, P.M. Boerrichter, M. Krijn, D. Feil and D. Sundholm, J. Mol. Struct. (Theochem), 133 (1985) 147.

19 M.P.C.M. Krijn and D. Feil, J. Chem. Phys., 85 (1986) 319.

20 J.D. Dunitz and P.J. Seiler, J. Am. Chem. Soc., 105 (1983) 7056.

21 R.F.W. Bader, Atoms in Molecules, Clarendon Press, Oxford, 1990.

22 N.L. Allen, C.G. West, D.L. Cooper, P.J. Grout and N.H. March, J.Chem. Phys., 83 (1985) 4562 .

23 R. Bader, Atoms in Molecules, Clarendon Press, Oxford, 1990, p. 277.

24 G. Moss and D. Feil, Acta Crystallogr., Sect. A, 37 (1981) 414.

A.J. Stone and M. Alderton, Mol. Phys., 56 (1985) 1047.

25 R. Bader, Atoms in Molecules, Clarendon Press, Oxford, 1990, p.130.

26 H. Bruning and $D$. Feil, in preparation.

27 F.L. Hirshfeld, Isr. J. Chem., 16 (1977) 198.

H. Bruning and D. Feil, J. Comput. Chem., 12 (1991) 1.

28 P. Coppens, J. Dam, S. Harkema, D. Feil, R. Feld, M.S. Lehman, R. Goddard, C. Kriiger, E. Hellner, H. Johansen, F.K. Larsen, T.F. Koetzle, R.K. McMullan, R.K. Maslen and E.D. Stevens, Acta Crystallogr., Sect. A, 40 (1984) 184.

29 R.F. Stewart, Acta Crystallogr. Sect. A, 32 (1976) 565.

F.L. Hirshfeld, Isr. J. Chem., 16 (1977) 226.

N. Hansen and P. Coppens, Acta Crystallogr., Sect. A, 34 (1978) 909.

30 P. Coppens, J. Phys. Chem., 93 (1989) 7979.

31 M.P.C.M. Krijn, H. Graafsma and D. Feil, Acta Crystallogr., Sect. B, 44 (1988) 609.

32 H. Bruning, in preparation.

33 W.L. Bragg, R.W. James and C.H. Bosanquet, Phil. Mag., 44 (1922) 435.

34 Z. Berkovitch-Yellin and L. Leiserowitz, J. Am. Chem. Soc., 102 (1980) 7677.

35 Z. Berkovitch-Yellin and L. Leiserowitz, J. Am. Chem. Soc., 104 (1982) 4052.

36 Z. Berkovitch-Yellin, J. Am. Chem. Soc., 107 (1985) 8239.

37 B. Weiner and Y. Öhrn, J. Phys. Chem., 91 (1987) 563.

E.S. Boek, D. Feil, W.J. Briels and P. Bennema, J. Cryst. Growth, 114 (1991) 389. 